\title{
Convex Optimization for Joint Expansion Planning of Natural Gas and Power Systems
}

\author{
Conrado Borraz-Sánchez \\ LANL, USA \\ conradob@lanl.gov \\ Seth Blumsack \\ The Pennsylvania State University, USA \\ sethb@psu.edu
}

\author{
Russell Bent \\ LANL, USA \\ rbent@lanl.gov \\ Hassan Hijazi \\ NICTA, Australia \\ Hassan.Hijazi@nicta.com.au
}

\author{
Scott Backhaus \\ LANL, USA \\ backhaus@lanl.gov \\ Pascal van Hentenryck \\ University of Michigan, USA \\ pvanhent@umich.edu
}

\begin{abstract}
Within the energy sector, two of the most tightly coupled systems are natural gas and electric power. The recent advent of cheap gas extraction technologies have only driven these systems more tightly together. Despite their interconnections, in many areas of the world these systems are operated and managed in isolation. This separation is due to a number of reasons and challenges, ranging from technological (problems involving connected systems are difficult to solve) to political and commercial (prevention of monopolies, lack of communication, market forces, etc.). However, this separation can lead to a number of undesirable outcomes, such as what the northeastern United States experienced during the winter of 2013/2014. In this paper, we develop approaches to address the technological reasons for separation. We consider the problem of expanding and designing coupled natural gas and electric power systems to meet increased coincident demand on both systems. Our approach utilizes recent advances in convex modeling of gas and power systems to develop a computationally tractable optimization formulation.
\end{abstract}

\section{Introduction}

In many parts of the world, the advent of cheap natural gas extraction technologies has led to record low prices for natural gas. This has led to rapid shifts in the fuel mixes of generator fleets in electric power systems. However, unlike other fossil fuels, where fuel is procured days in advance and/or there is limited competition for the fuel, natural gas is procured on much faster time scales and is exposed to significant sources of competition from other sectors (such as heating). As a result, while cheap natural gas has driven the price of electricity down, it has also increased fuel supply risk within power systems. Recent experiences in the northeast United States, particularly during the winter of 2013/14 have highlighted this risk. Record cold temperatures directed gas supply to heating applications, resulting in difficulties in procuring fuel for gas-fired generators, and leaving power systems scrambling for alternate sources of power. In this case, there were no blackouts due to generation shortages, but there were significant economic consequences [1]. A general discussion on economic consequences of gas volatility can also be found in [2].

The risk associated with increased reliance on natural gas is influenced by a number of factors, one of which is the inherent separation in management and operation of gas and power systems. This separation exists for a number of reasons.
From a technological perspective, problems that join both systems are computationally very difficult and discourage joint management. Second, in many places the sectors are prohibited from coordinating activities or disjoint market structures discourage coordination.

One area of separation that could benefit from coordination is expansion planning. First, natural gas and electric power systems are undergoing expansion - new natural gas pipelines are being built to connect new supplies to existing markets and power systems are continuing to shift away from coal and towards natural gas. These system expansions are not well-coordinated and represent an additional pathway for fuel supply risk reduction within the power system. Second, some of the factors influencing the problems experienced in the northeastern United States included a lack of pipeline capacity to deliver fuel to generators that needed it and lack of power transfer capacity to deliver power from areas that had excess fuel capacity. Had the networks been designed with additional capacity in anticipation of their joint needs, such a situation may have been avoided.

In this paper, we develop a new joint expansion planning model for gas and power to address this problem. Fig. 1 shows a schematic of the electricity and natural gas coupling infrastructure which is based on illustrations taken from [3], [4], [5], [6]. We focus on solving the computational challenges associated with this problem and we base our approach on recent advances in convex relaxations developed for power and gas systems [7], [8]. This paper does not address the issues associated with implementing the solutions of this model. Our aim is to develop a modeling framework and test system to explore the space of solutions, to help policy makers and regulators identify the gains from joint infrastructure planning so that they might find mechanisms to encourage their development.

The key contributions of the paper include:

1) A tractable relaxation of joint gas-electric physics for use in expansion planning.

2) A realistic large-scale joint gas-electric system for use by the community.

3) A demonstration of the approach on coincident peak demand scenarios.

Literature Review The literature on combined gas-electricity expansion planning problems is relatively sparse [3], [4], [5], [6], [9]. In contrast, there are a number of papers that jointly 


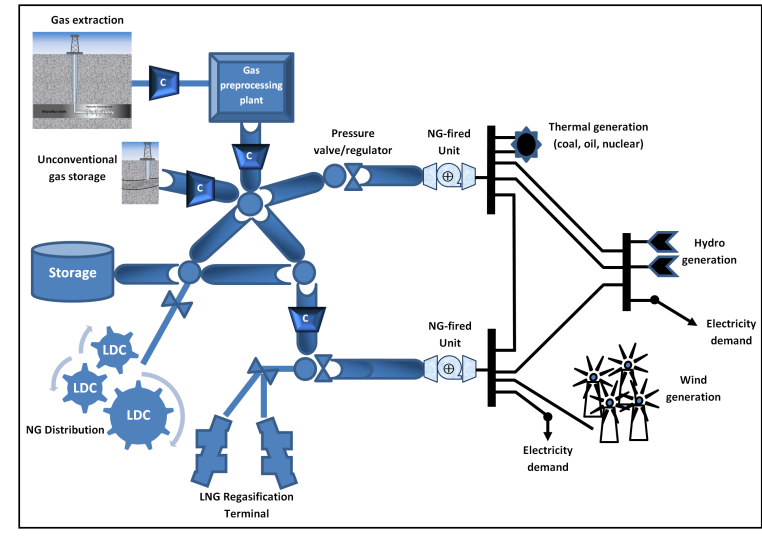

Figure 1: Schematic of the electricity and natural gas coupling infrastructure.

optimize the operations of power and gas systems [10], [11], [12], [13], [14]. Unlike this paper, much of the state-of-theart on combined problems relies on capacity models or linear approximations of the underlying physics in the system. For example, references [3], [4], [5], [9], [10], [11], [12], [13], [14], [15] use the linearized DC model of power systems. We next briefly review those papers that have considered joint gas-power expansion planning problems and those papers that consider joint operations models that are the most similar to the models of this paper. Comprehensive literature surveys on integrated natural gas and electricity operation planning can be found in, e.g., [5], [6].

First, reference [9] presents a model that minimizes operational and expansion costs on both the gas and electricity systems. Their model includes expansions of generators, pipes, compressors and storage facilities. Power flows are modeled with the DC-OPF and gas flows are modeled with the Panhandle A equations. Their problem is a multi-stage problem.

Reference [3] develops a bi-objective MILP model to solve generation expansion planning between the gas and electricity systems. They use transportation models for the systems. The objective minimizes investment cost and maximizes reliability with engineered economic functions. The authors illustrate their approach on a 6-bus test network.

Reference [16] proposes a multi-stage approach for solving the gas and electricity expansion planning problem. The model is formulated as a mixed integer linear program and solved via cplex. A single-pipeline and a two bus power system is presented as a study case. They extend their work in [15] with a case study based on the Brazilian integrated gas/electricity system. Their main result emphasizes the importance of natural gas storage facilities in presence of hydro power because of the risks involved with water flow uncertainty. [14] presents a model of joint gas and power dispatch without expansion planning. However, this is one of the few papers that uses the full non-linear steady-state models of power and gas. The authors developed an evolutionary algorithm where the feasibility and fitness of dispatch solutions are evaluated using numerical simulation methods to solve the power and gas equations. Their test system is based on combining the IEEE14 power system with the Belgian gas network and is the basis for one of our test systems.

Reference [10] presents a nonlinear model that maximizes social welfare of gas and electricity systems. The systems are solved independently with a two-phase algorithm. The algorithm first solves the electric power system, and then uses this solution as input for solving the natural gas system. A primal-dual interior-point method is applied to a variation of the IEEE- 5 bus system.

Reference [11] presents a combined gas and electricity model where the DC model is used to represent the power system. They include a multi-time period feature that is based on a quasi-steady state model of gas. The model is used to calculate line-pack on daily time scales. ODEs are derived from a finite difference scheme that is used to represent the temporal and spatial derivatives of the original PDEs of the gas model. A sequential linear programming approach is used to solve the problem.

In an operational context, [12] develops a MILP model to solve a security-constrained power and natural-gas flow problem. The power system is based on a DC-OPF model and the gas system is modeled with a piece-wise linear approximation. They study a model that combines the IEEE 24-bus system and with a modified Belgian high-calorific gas network. Reference [13] presents an approach that solves the gas flow network problem under unit commitment conditions of the power system.

Finally, there are number of papers that motivate the need for joint power and gas expansion solutions. For example, [4] analyzes cost estimations for the Marcellus pipeline expansions and their impact to the Midwest Independent Transmission System Operator (MISO). Reference [6] also provides a survey of operations, planning, and market questions that need to be addressed within integrated natural gas and electricity problems. Reference [1] provides an interesting discussion of impacts to the PJM system when coincident peaks in gas and power consumption occurred in the winter of 2014.

The remainder of this paper is organized as follows: Section 2 describes the joint optimization model. Section 3 describes the test systems. Section 4 discusses some experimental results. We conclude with Section 5.

\section{Joint electricity and gas expansion model}

The framework of the combined electricity and gas expansion (CEGE) system consists of structured blocks that include physical, operational, contractual, design (On/Off) and coupling constraints imposed on both gas and electricity systems:

subject to:

$$
\min _{\Lambda, \omega}\left(\mathcal{C}_{\exp }(\Lambda)+\mathcal{G}_{m i x}(\omega)\right)
$$

\begin{tabular}{|c|c|}
\hline (Power grid constraints) & (Gas flow constraints) \\
\hline Power flow equations & Gas flow equations \\
\hline Line limits & Pressure bounds \\
\hline Voltage limits (AC) & Mass flow balance \\
\hline Generation bounds & Compressor ratio limits \\
\hline Coupling & nstraints \\
\hline
\end{tabular}


Table 1: Nomenclature of the natural gas (NG) pipeline system.

\begin{tabular}{|c|c|}
\hline Symbol & Definition \\
\hline $\mathbb{G}=\left(N^{g}, A^{g}\right)$ & NG system base configuration \\
\hline \multicolumn{2}{|l|}{ SETS: } \\
\hline$N^{g}$ & All nodes in $\mathbb{G}$ \\
\hline$A^{g}$ & All links joining a pair of nodes $(i, j)$ in $\mathbb{G}$ \\
\hline$T$ & Nodes with Gas-fired power plants, $T \subseteq N^{g}$ \\
\hline$A_{p}$ & Base pipelines, $A_{p} \subseteq A^{g}$ \\
\hline$A_{c}$ & Base compressors, $\bar{A}_{c} \subseteq A^{g}$ \\
\hline \multicolumn{2}{|l|}{ PARAMETERS: } \\
\hline W & Pipeline resistance (Weymouth) factor \\
\hline$U$ & Pipeline maximum capacity (contractual) \\
\hline$\underline{\alpha}, \bar{\alpha}$ & Compression limits at compressor stations \\
\hline$D$ & Nodal fixed consumption \\
\hline$\underline{D}, \bar{D}$ & Nodal consumption limits \\
\hline$\underline{S}, \bar{S}$ & Nodal production limits \\
\hline $\bar{\pi}, \bar{\pi}$ & Nodal pressure limits (squared) \\
\hline \multicolumn{2}{|l|}{ VARIABLES: } \\
\hline$\pi$ & Nodal pressure (squared) \\
\hline$x$ & Gas flow on pipelines and compressors \\
\hline$\lambda$ & auxiliary relaxation variable \\
\hline$d$ & Nodal gas consumption \\
\hline$s$ & Nodal gas production \\
\hline$y^{+}$ & Forward (positive) flow direction (Binary) \\
\hline$y^{-}$ & Reverse (negative) flow direction (Binary) \\
\hline
\end{tabular}

where the functions $\mathcal{C}_{\text {exp }}(\Lambda)$ and $\mathcal{G}_{\text {mix }}(\omega)$ in the objective represent, respectively, capital expenditures (upgrades) and generation costs, i.e.:
$\mathcal{C}_{\text {exp }}=$
(Capital expenditures)
EP generation capital +
EP transmission capital +
NG transmission capital +
NG compression capital

$$
\begin{gathered}
\underbrace{(\text { Generation mix) }}_{\begin{array}{c}
\mathcal{G}_{\text {mix }}= \\
\text { min } \sum_{i} \sum_{t} C\left(g_{i}^{t}\right)
\end{array}} \\
\text { where: } \begin{array}{l}
C\left(g_{i}^{t}\right)=\text { cost of power } \\
\text { generation }(g) \text { of fuel } \\
\text { type } t \text { at bus } i
\end{array}
\end{gathered}
$$

The CEGE problem is a mixed-integer non-linear program (MINLP) that is very difficult to solve. In the next sections, we describe the full problem in detail and discuss recent high quality convex relaxations developed in the literature that we use to convert the problem into a computationally tractable optimization. Unless otherwise noted, parameters and variables are indexed over the sets of base network components and expansion candidates. The nomenclature is presented in Tables 1, 2, and 3 .

\subsection{Relaxations for bilinear terms}

In this model we make heavy use of bilinear relaxations introduced by McCormick in [17], [18] and widely applied in [8], [19], [20]. Intuitively, this relaxation formulates convex envelopes for bilinear terms. Given a product of two variables, $x y$, the relaxation is denoted by $\check{w} \in\langle x, y\rangle^{\mathcal{M}_{c}}$, which represents the following set of linear constraints:

\begin{tabular}{|c|c|}
\hline Symbol & Definition \\
\hline $\mathbb{E}=\left(N^{e}, A^{e}\right)$ & EP system base configuration \\
\hline \multicolumn{2}{|l|}{ SETS: } \\
\hline$N^{e}$ & All nodes in $\mathbb{E}$ (electrical buses) \\
\hline$A^{e}$ & All transmission lines in $\mathbb{E}$ \\
\hline$\Omega$ & Power generators \\
\hline$\Omega^{g}$ & Gas-fired power generators, $\Omega^{g} \subseteq \Omega$ \\
\hline$\Omega^{e}$ & Non-gas-fired power generators, $\bar{\Omega}^{e} \subseteq \Omega$ \\
\hline$\Gamma$ & Generators of a bus \\
\hline \multicolumn{2}{|l|}{ PARAMETERS: } \\
\hline MVABase & Base apparent power \\
\hline$\delta_{0}$ & Slack/swing bus \\
\hline$P L$ & Nodal active power load \\
\hline$Q L$ & Nodal reactive power load \\
\hline$C_{0}, C_{1}, C_{2}$ & Power generation cost coefficients \\
\hline$\underline{\underline{V}}, \bar{V}$ & Nodal voltage magnitude limits \\
\hline$\underline{P_{G}}, \overline{P_{G}}$ & Active power generation limits \\
\hline$\overline{Q_{G}}, \overline{Q_{G}}$ & Reactive power generation limits \\
\hline $\bar{\theta}^{u}$ & Phase angle difference bound \\
\hline$X$ & Line reactance (reactive power loss) \\
\hline$R$ & Line resistance(active power loss) \\
\hline$\tilde{S}$ & Thermal limit/capacity of line \\
\hline$g$ & Line conductance, $=\frac{R}{\left(X^{2}+R^{2}\right)}$ \\
\hline$b$ & Line susceptance, $=\frac{X}{X^{2}+R^{2}}$ \\
\hline \multicolumn{2}{|l|}{ VARIABLEs: } \\
\hline$\hat{p}$ & Active power flow on lines \\
\hline$\hat{q}$ & Reactive power flow on lines \\
\hline$p g$ & Active power generation at generators \\
\hline$q g$ & Reactive power generation at generators \\
\hline$v$ & Nodal voltage magnitude \\
\hline$\theta$ & Nodal phase angle \\
\hline$\check{v}$ & auxiliary relaxation variable \\
\hline$\breve{w c}$ & auxiliary relaxation variable \\
\hline$\check{w s}$ & auxiliary relaxation variable \\
\hline
\end{tabular}

$$
\langle x, y\rangle^{\mathcal{M}_{c}} \equiv\left\{\begin{array}{l}
\check{w} \geq \mathbf{x}^{l} y+\mathbf{y}^{l} x-\mathbf{x}^{l} \mathbf{y}^{l} \\
\check{w} \geq \mathbf{x}^{u} y+\mathbf{y}^{u} x-\mathbf{x}^{u} \mathbf{y}^{u} \\
\check{w} \leq \mathbf{x}^{l} y+\mathbf{y}^{u} x-\mathbf{x}^{l} \mathbf{y}^{u} \\
\check{w} \leq \mathbf{x}^{u} y+\mathbf{y}^{l} x-\mathbf{x}^{u} \mathbf{y}^{l}
\end{array}\right.
$$

where $\left[\mathbf{x}^{l}, \mathbf{x}^{u}\right]$ and $\left[\mathbf{y}^{l}, \mathbf{y}^{u}\right]$ represent the minimum and maximum bounds on $x$ and $y$, respectively. Note that when $x$ or $y$
Table 2: Nomenclature of the electric power (EP) grid.

Table 3: Nomenclature of the design and coupling attributes.

\begin{tabular}{cll} 
Symbol & & Definition \\
\cline { 1 - 1 } SETS: & & Pipeline expansion candidates \\
$\Lambda_{P}$ & & Compressor expansion candidates \\
$\Lambda_{C}$ & & Power generator expansion candidates \\
$\Lambda_{G}$ & & Transmission line expansion candidates \\
$\Lambda_{T}$ & & The generator associated with a natural gas node \\
$\gamma$ & & \\
PARAMETERS: & & Pipeline installation cost \\
$c^{p}$ & & Compressor installation cost \\
$c^{c}$ & & Electric transmission line installation cost \\
$c^{t}$ & & Power generator installation cost \\
$c^{g}$ & Heat rate curve coefficients \\
$H_{1}, H_{2}, H_{3}$ & Pipeline candidate is added; 0 otherwise \\
$($ BINARY) VARIABLES equal to 1 if: & Compressor candidate is added; 0 otherwise \\
$z^{p}$ & & Transmission line candidate is added; 0 otherwise \\
$z^{c}$ & Power generator candidate is added; 0 otherwise \\
$z^{t}$ & &
\end{tabular}

are binary variables this relaxation is tight. We also make use of the following relaxation for quadratic terms of the form $v^{2}$ :

$$
\left\langle v^{2}\right\rangle^{\mathcal{R}} \equiv\left\{\begin{array}{l}
\check{v} \geq v^{2} \\
\check{v} \leq\left(\mathbf{v}^{u}+\mathbf{v}^{l}\right) v-\mathbf{v}^{u} \mathbf{v}^{l}
\end{array}\right.
$$

where $\left[\mathbf{v}^{l}, \mathbf{v}^{u}\right]$ represents the bounds on $v$.

\subsection{Objective function}

The objective function of the CEGE model minimizes the total cost incurred by power generation dispatch and expected upgrade costs of the CEGE system. The primary operational 
cost for the gas transmission system is compression, which is small relative to other costs in this model and is ignored. Formally, the objective takes the form of Eq. (1).

$$
\begin{gathered}
\min \left[\beta * \left(\sum_{i \in \Omega^{e}}\left(C_{2_{i}} p g_{i}^{2}+C_{1_{i}} p g_{i}+C_{0_{i}}\right)\right.\right. \\
\left.+\sum_{i \in T}\left(C_{2_{i}} d_{i}^{2}+C_{1_{i}} d_{i}+C_{0_{i}}\right)\right)+(1-\beta)\left(\sum_{i \in \Lambda_{P}} c_{i}^{p} z_{i}^{p}+\right. \\
\left.\left.\sum_{i \in \Lambda_{C}} c_{i}^{c} z_{i}^{c}+\sum_{i \in \Lambda_{T}} c_{i}^{t} z_{i}^{t}+\sum_{i \in \Lambda_{G}} c_{i}^{g} z_{i}^{g}\right)\right]
\end{gathered}
$$

The first term calculates the total cost of power generation from non-gas fired plants. The second term calculates the cost of power generation from gas-fired plants. This cost is determined directly from the amount of gas that is consumed (i.e., the gas-grid coupling). While this separation is unnecessary, such a separation (as is discussed later) helps to ensure that our relaxation on the quadratic constraint that relates gas consumption to power produced is tight. The remaining terms model the costs for upgrading pipelines, compressors, transmission lines and generators, respectively. The $0 \leq \beta \leq 1$ term is used to control the relative weighting between expansion costs and operating costs.

\subsection{Gas flow model}

The gas flow model is based on the formulation proposed in [7]. It uses integer variables to denote the flow direction of the flow in each pipe. The first set of constraints of the CEGE model handles the physics associated with gas systems and is expressed as follows:

$$
\begin{aligned}
& \sum_{i j \in A^{g}} x_{i j}-\sum_{j i \in A^{g}} x_{j i}=s_{i}-d_{i}-D_{i}, \quad \forall i \in N^{g}, \\
& y_{i j}^{+}+y_{i j}^{-}=1 \text {, } \\
& z_{i j}^{p}\left(y_{i j}^{+}-y_{i j}^{-}\right)\left(\pi_{i}-\pi_{j}\right)=W_{i j} x_{i j}^{2}, \quad \forall(i, j) \in A_{p} \text {, } \\
& -\left(1-y_{i j}^{+}\right) \sum_{i \in N^{g}} \bar{S}_{i} \leq x_{i j}, \\
& \forall(i, j) \in A^{g},(5) \\
& x_{i j} \leq\left(1-y_{i j}^{-}\right) \sum_{i \in N^{g}} \bar{S}_{i}, \\
& \forall(i, j) \in A^{g},(6) \\
& \left(1-y_{i j}^{+}\right) \underline{\pi} \leq \pi_{i}-\pi_{j} \leq\left(1-y_{i j}^{-}\right) \bar{\pi}, \quad \forall(i, j) \in A_{p},(7) \\
& \pi_{i} \underline{\alpha}_{i j}-\left(2-y_{i j}^{+}-z_{i j}^{c}\right)\left(\bar{\pi}_{i} \underline{\alpha}_{i j}-\underline{\pi}_{j}\right) \leq \pi_{j}, \quad \forall(i, j) \in A_{c},(8) \\
& \pi_{j} \leq \pi_{i} \bar{\alpha}_{i j}+\left(2-y_{i j}^{+}-z_{i j}^{c}\right)\left(\bar{\pi}_{j}-\underline{\pi}_{i} \bar{\alpha}_{i j}\right), \quad \forall(i, j) \in A_{c},(9) \\
& \pi_{j} \underline{\alpha}_{i j}-\left(2-y_{i j}^{-}-z_{i j}^{c}\right)\left(\bar{\pi}_{j} \underline{\alpha}_{i j}-\underline{\pi}_{i}\right) \leq \pi_{i}, \quad \forall(i, j) \in A_{c}(10) \\
& \pi_{i} \leq \pi_{j} \bar{\alpha}_{i j}+\left(2-y_{i j}^{-}-z_{i j}^{c}\right)\left(\bar{\pi}_{i}-\underline{\pi}_{j} \bar{\alpha}_{i j}\right), \quad \forall(i, j) \in A_{c}(11) \\
& \underline{D}_{i} \leq d_{i} \leq \bar{D}_{i}, \quad \forall i \in N^{g} \text {, } \\
& \underline{S}_{i} \leq s_{i} \in \bar{S}_{i} \\
& \forall i \in N^{g} \text {, } \\
& \underline{\pi}_{i} \leq \pi_{i} \in \bar{\pi}_{i}, \\
& \forall i \in N^{g} \text {, } \\
& y_{i j}^{+}, y_{i j}^{-} \in\{0,1\} \text {, } \\
& \forall(i, j) \in A^{g}(.15)
\end{aligned}
$$

and the pipe's resistance characteristics (typically a function of the pipe's diameter, length, and other physical properties). Eqs. (5) and (6) ensure that flow directionality is tied to the sign of the flow. Eqs. (8)-(11) ensure that the compression (pressure boost) falls within the compression ratio limits of the compressors. Finally, Eqs. (12)-(14) ensure that consumption, production, and pressure fall within limits.

In this formulation, the only source of non-convexity is Eq. (4). Using recent results established by [7], part of the non-convexity can be eliminated by substituting (tightly) $\lambda_{i j}$ for $\left(y_{i j}^{+}-y_{i j}^{-}\right)\left(\pi_{i}-\pi_{j}\right)$, where $\lambda_{i j} \in\left\langle y_{i j}^{+}-y_{i j}^{-}, \pi_{i}-\pi_{j}\right\rangle^{M c}$. This replaces Eq. (4) with

$$
z_{i j}^{p} \lambda_{i j}=W_{i j} x_{i j}^{2}
$$

which is still non convex. However, [7] has shown that the second-order cone relaxation

$$
z_{i j}^{p} \lambda_{i j} \geq W_{i j} x_{i j}^{2}
$$

is very often tight in practice for expansion planning problems and we use this formulation here. [7] also includes a number of provably valid cuts on the integer variables that improve performance as well as models of valves, resistors, and short pipes that we also use. However, for simplicity and brevity, we omit those details here.

\subsection{AC-power flow model}

The second set of constraints added to the CEGE model describes the physics and operational decision points for the power system. This model is based on the seminal second order cone relaxations derived by [8]. For notional simplicity and brevity, we omit the terms and constraints related to line losses, currents, and shunt compensation. The networks in our experimental results include some of these features and interested readers are invited to consult [8] for the details on how to incorporate these features.

$$
\begin{array}{cl}
\theta_{\delta_{0}}=0, \sum_{j \in \Gamma_{i}} p g_{j}-P L_{i}=\sum_{(i, j) \in A^{e}} \hat{p}_{i j}, & \forall i \in N^{e}, \\
\sum_{j \in \Gamma_{j}} q g_{j}-Q L_{i}=\sum_{(i, j) \in A^{e}} \hat{q}_{i j}, & \forall i \in N^{e}, \quad \text { (19) } \\
\hat{p}_{i j}=g_{i j} v_{i}^{2}-g_{i j} v_{i} v_{j} \cos \left(\theta_{i}-\theta_{j}\right) & \\
-b_{i j} v_{i} v_{j} \sin \left(\theta_{i}-\theta_{j}\right), & \forall(i, j) \in A^{e},(21) \\
\hat{q}_{i j}=-b_{i j} v_{i}^{2}+b_{i j} v_{i} v_{j} \cos \left(\theta_{i}-\theta_{j}\right) & \\
-g_{i j} v_{i} v_{j} \sin \left(\theta_{i}-\theta_{j}\right), & \forall(i, j) \in A^{e},(22) \\
\hat{p}_{i j}^{2}+\hat{q}_{i j}^{2} \leq\left|\tilde{S}_{i j}\right|^{2}, & \forall(i, j) \in A^{e},(23) \\
-\theta_{i j}^{u} \leq \theta_{i}-\theta_{j} \leq \theta_{i j}^{u}, & \forall(i, j) \in A^{e},(24) \\
z_{i}^{g} \frac{P_{G_{i}} \leq p g_{i} \leq z_{i}^{g}}{P_{G i}}, & \forall i \in \Omega, \\
z_{i}^{g} \underline{Q}_{G_{i}} \leq q g_{i} \leq z_{i}^{g} \overline{Q_{G}}, & \forall i \in \Omega,
\end{array}
$$

Eq. (18) sets a swing bus reference phase angle to 0 . Kirchhoff's Current Law equations (flow conservation) for real and reactive power are described in Eqs. (19) and (20),

to the (squared) pressure difference at either end of the pipe 
respectively. The real and reactive power flow on each line (Ohm's Law) are stated with Eqs. (21) and (22). Eqs. (23) and (24) state operational limits of, respectively, the thermal line loading and the phase angle difference $\left|\theta_{i}-\theta_{j}\right|$. Finally, Eqs. (25)-(27) impose active power generation, reactive power generation and voltage magnitude limits at each bus.

The main difficulties in this second set of constraints (ACpower flow model) arise from the non-convexities in Eqs. (21) and (22). In this paper we use the result of [8] to relax these equations to the following set of convex constraints:

$$
\begin{aligned}
\hat{p}_{i j}=g_{i j} \check{v}_{i j}-g_{i j} \check{w} c_{i j}-b_{i j} \check{w} s_{i j}, & \forall(i, j) \in A^{e}, \\
\hat{q}_{i j}=-b_{i j} \check{v}_{i j}+b_{i j} \check{w} c_{i j}-g_{i j} \check{w} s_{i j}, & \forall(i, j) \in A^{e}, \\
\check{c} s_{i j} \in\left\langle\cos \left(\theta_{i}-\theta_{j}\right)\right\rangle^{\mathcal{R}}, & \forall(i, j) \in A^{e}, \\
\check{s}_{i j} \in\left\langle\sin \left(\theta_{i}-\theta_{j}\right)\right\rangle^{\mathcal{R}}, & \forall(i, j) \in A^{e}, \\
\check{v}_{i} \in\left\langle v^{2}\right\rangle^{\mathcal{R}}, & \forall i \in N^{e}, \\
\check{w}_{i j} \in\left\langle v_{i}, v_{j}\right\rangle^{\mathcal{M}_{c}}, & \forall(i, j) \in A^{e}, \\
\check{w} c_{i j} \in\left\langle\check{w}_{i j}, \check{c s}_{i j}\right\rangle^{\mathcal{M}_{c}}, & \forall(i, j) \in A^{e}, \\
\check{w} s_{i j} \in\left\langle\check{w}_{i j}, \check{s}_{i j}\right\rangle^{\mathcal{M}_{c}}, & \forall(i, j) \in A^{e},
\end{aligned}
$$

where

$$
\langle\cos (\theta)\rangle^{\mathcal{R}} \equiv\left\{\begin{array}{l}
\check{c s} \geq \cos \left(\theta^{u}\right) \\
\check{c s} \leq 1-\frac{1-\cos \left(\theta^{u}\right)}{\left(\theta^{u}\right)^{2}} \theta^{2}
\end{array}\right.
$$

and

$$
\langle\sin (\theta)\rangle^{\mathcal{R}} \equiv\left\{\begin{array}{l}
\check{s} \geq \cos \left(\theta^{u} / 2\right)\left(\theta-\theta^{u} / 2\right)-\sin \left(\theta^{u} / 2\right) \\
\check{s} \leq \cos \left(\theta^{u} / 2\right)\left(\theta-\theta^{u} / 2\right)+\sin \left(\theta^{u} / 2\right)
\end{array}\right.
$$

We next introduce on/off constraints to model whether or not lines are built (these are analogous to the switching variables of [8]). We first define some large constants:

$$
\begin{aligned}
& \overline{M_{\theta}}=\sum_{i j \in E^{e}} \theta^{u} \\
& \underline{M_{\theta}}=\sum_{i j \in E^{e}}-\theta^{u} \\
& \overline{V V}_{i j}=\bar{V}_{i} * \bar{V}_{j} \\
& \underline{V V_{i j}}=\underline{V}_{i} * \underline{V}_{j} \\
& \overline{W R}_{i j}=\overline{V V}_{i j} \\
& \underline{W R_{i j}}=\overline{V V}_{i j} * \cos \left(-\theta^{u}\right) \\
& \overline{W I}_{i j}=\overline{V V}_{i j} * \sin \left(\theta^{u}\right) \\
& \underline{W I}_{i j}=\overline{V V}_{i j} * \sin \left(-\theta^{u}\right)
\end{aligned}
$$

The expansion planning model is now now stated as:

$$
\begin{aligned}
& \underline{V}_{i}^{2} z_{i j}^{t} \leq \check{v}_{i j} \leq \bar{V}_{i}^{2} z_{i j}^{t}, \quad \forall(i, j) \in E^{e},(44) \\
& \check{v}_{i}-\left(1-z_{i j}^{t}\right) \bar{V}_{i}^{2} \leq \check{v}_{i j} \leq \check{v}_{i}-\left(1-z_{i j}^{t}\right) \underline{V}_{i}^{2} \quad \forall(i, j) \in E^{e},(45) \\
& \theta_{i}-\theta_{j} \geq-\theta_{i j}^{u} z_{i j}^{t}+\underline{M_{\theta}}\left(1-z_{i j}^{t}\right), \quad \forall(i, j) \in E^{e}, \\
& \theta_{i}-\theta_{j} \leq \theta_{i j}^{u} z_{i j}^{t}+\overline{M_{\theta}}\left(1-z_{i j}^{t}\right), \quad \forall i \in N^{e}, \\
& \sin \left(-\theta_{i j}^{u}\right) z_{i j}^{t} \leq \check{s i}_{i j} \leq \sin \left(\theta_{i j}^{u}\right) z_{i j}^{t}, \quad \forall(i, j) \in E^{e}, \\
& \check{s i}_{i j}-\cos \left(\frac{\theta_{i j}^{u}}{2}\right)\left(\theta_{i}-\theta_{j}\right) \leq \\
& \left(\sin \left(\frac{\theta_{i j}^{u}}{2}\right)-\cos \left(\frac{\theta_{i j}^{u}}{2}\right) \frac{\theta_{i j}^{u}}{2}\right) z_{i j}^{t} \\
& +\left(\cos \left(\frac{\theta_{i j}^{u}}{2}\right) \overline{M_{\theta}}\right)\left(1-z_{i j}^{t}\right), \quad \forall(i, j) \in E^{e},(49) \\
& -\check{s} i_{i j}+\cos \left(\frac{\theta_{i j}^{u}}{2}\right)\left(\theta_{i}-\theta_{j}\right) \leq \\
& \left(\sin \left(\frac{\theta_{i j}^{u}}{2}\right)-\cos \left(\frac{\theta_{i j}^{u}}{2}\right) \frac{\theta_{i j}^{u}}{2}\right) z_{i j}^{t} \\
& +\left(\cos \left(\frac{\theta_{i j}^{u}}{2}\right) \overline{M_{\theta}}\right)\left(1-z_{i j}^{t}\right), \quad \forall(i, j) \in E^{e},(50) \\
& \cos \left(-\theta_{i j}^{u}\right) z_{i j}^{t} \leq \check{c s_{i j}} \leq z_{i j}^{t}, \quad \forall(i, j) \in E^{e},(51) \\
& \check{c} s_{i j}+\frac{1-\cos \left(\theta_{i j}^{u}\right)}{\left(\theta_{i j}^{u}\right)^{2}}\left(\theta_{i}-\theta_{j}\right)^{2} \leq z_{i j}^{t} \\
& +\left(1-z_{i j}^{t}\right)\left(\frac{{\overline{M_{\theta}}}^{2}\left(1-\cos \left(\theta_{i j}^{u}\right)\right)}{\left(\theta_{i j}^{u}\right)^{2}}\right), \quad \forall(i, j) \in E^{e}, \\
& \underline{V V}_{i j} z_{i j}^{t} \leq \check{w}_{i j} \leq \overline{V V}_{i j} z_{i j}^{t}, \quad \forall(i, j) \in E^{e}, \\
& \underline{W R}_{i j} z_{i j}^{t} \leq \check{w}_{i j} \leq \overline{W R}_{i j} z_{i j}^{t}, \quad \forall(i, j) \in E^{e}, \\
& \underline{W I}_{i j} z_{i j}^{t} \leq \check{w} s_{i j} \leq \overline{W I}_{i j} z_{i j}^{t}, \quad \forall(i, j) \in E^{e} .
\end{aligned}
$$

Eqs. (44) and (45) state the power flow disjunction to account for the off-configuration of the line where $\hat{p}=\hat{q}=0$. Eqs. (46)-(48) imposes bounds on the phase angle difference and the convex sine relaxation, respectively. Eqs. (49)(50) and (51)-(52) state the off-restrictions of the convex envelopes on the sine and cosine constraints, respectively. Finally, Eqs. (53)-(55) forces relaxation variables to 0 when transmission lines are not built. McCormick Eqs. (33), (34), and (35) are also modified so that they are only active when the lines are built (see [8]).

\subsection{Coupling and design constraints}

The coupling constraint between the gas and electricity network systems is given by the inequality:

$$
d_{i} \geq H_{1}^{\gamma_{i}}+H_{2}^{\gamma_{i}} p g_{\gamma_{i}}+H_{3}^{\gamma_{i}} p g_{\gamma_{i}}^{2}, \forall i \in T
$$

which relaxes the equality condition which is non-convex. Eq. (56) imposes the coupling constraint on the gas-fired power plants and gas terminals where the power grid and the gas system share a common location. This constraint defines the heat rate curve based on a gas consumption $d$ in $\mathrm{mmBTU} / \mathrm{h}$, power output $p g$ in $\mathrm{MW}$, and gas fuel rate coefficients $H_{1}^{i}, H_{2}^{i}$, and $H_{3}^{i}$ at generator $\gamma_{i}$ for peaking and shoulder plants. Note that the $H_{0}$ cost coefficient can be viewed as a no-load cost, i.e., a spinning cost. As mentioned, this constraint is expressed as an inequality in order to preserve 
convexity. In general, this constraint is tight due to the cost associated with consuming gas. When it is not tight, the constraint can be interpreted physically as throwing gas away. This can occur to maintain feasibility of pressure constraints and indicates the need for storage or other pressure regulating controls in this part of the system.

Finally, Eqs. (57)-(60) below state that the base network components of the CEGE system remain unchanged $(z=1)$ during the optimization process:

$$
\begin{array}{ll}
z_{i j}^{p}=1, & \forall(i, j) \in A^{p} \backslash \Lambda_{P}, \\
z_{i j}^{c}=1, & \forall(i, j) \in A^{c} \backslash \Lambda_{C}, \\
z_{i j}^{t}=1, & \forall(i, j) \in E^{e} \backslash \Lambda_{T}, \\
z_{i}^{g}=1, & \forall i \in \Omega \backslash \Lambda^{g} .
\end{array}
$$

\section{Test System Descriptions}

In this paper we utilize two integrated gas-grid test systems for demonstrating the performance of our approach. First we adopt the model of [14] that combines the IEEE 14 test system with the Belgian natural gas network (see Fig.2). Table 4 shows the technical details of proposed expansion plans for the underlying CEGE system.

Table 4: Technical details of the combined electricity-gas expansion system based on the integration of the IEEE_14-bus power system and the Belgian gas transmission network.

\begin{tabular}{rrrrr}
\hline \hline IEEE_14-bus & & \multicolumn{2}{c}{ Belgian system } \\
\cline { 5 - 5 } \cline { 5 - 5 } Buses & 14 & & Nodes & 22 \\
Generators & 5 & & Compressors & 3 \\
Base lines & 80 & & Base pipelines & 24 \\
Candidate lines & 80 & & Candidate pipes & 24 \\
Gas-fired generators & 2 & & Sources & 6 \\
& & & Terminals & 10 \\
\cline { 5 - 6 } & &
\end{tabular}

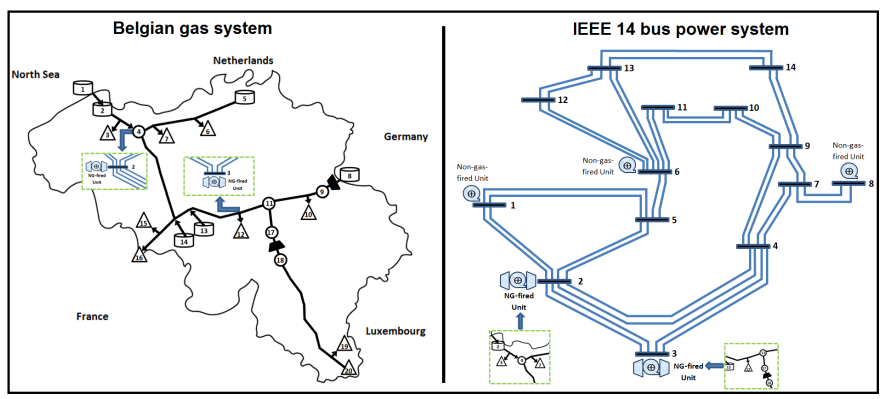

Figure 2: Schematics of the integrated IEEE 14 bus and Belgiangas network where gas-fired power generators 2 and 3 in the power grid are coupled with gas terminals 4 and 12 in the gas system.

Second, we develop a new test system for integrated power grid and natural gas pipeline simulation studies and implement the test system assuming a coincident peak case for both natural gas and electricity demand (similar in nature to what was observed in the U.S. northeast in the winter of 2013/14). Our test system is based on the architecture of the northeastern U.S. power grid and natural gas pipeline infrastructure. It captures, roughly, the geographic area from Pennsylvania northeast to New England, and incorporates natural gas and electrical interconnections with the Canadian provinces of Ontario, Quebec and Nova Scotia. A schematic of the test system is shown in Fig. 3. Table 5 shows the network components of the CEGE system for the New England case.

Table 5: Network components of the integrated IEEE_36-bus and Pennsylvania-To-Northeast New England expansion system.

\begin{tabular}{rrrrr}
\hline \hline IEEE_36-bus & & & \multicolumn{2}{c}{ Penn-To-Northeast system } \\
${$\cline { 5 - 5 }$} }$ & 36 & & Nodes & 125 \\
Base lines & 121 & & Compressors & 29 \\
Candidate lines & 121 & & Candidate pipes & 93 \\
Gas-fired generators & 36 & & Sources & 12 \\
Other-fuel generators & 216 & & Terminals & 37 \\
& & & Control valves & 21 \\
\cline { 5 - 6 }
\end{tabular}

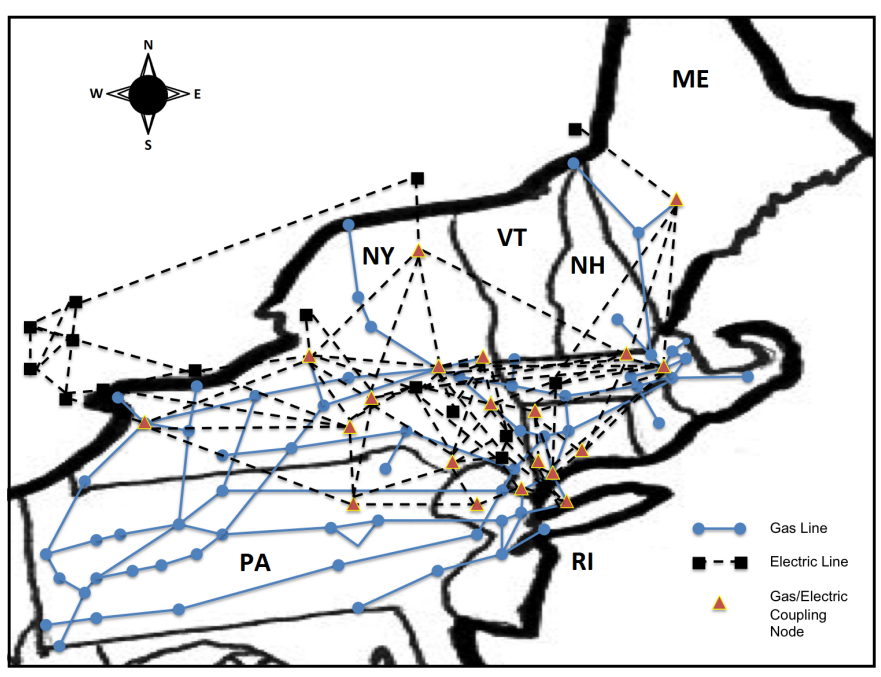

Figure 3: Network structure schematic for the New England (CEGE) model: The 36-bus grid system and the Pennsylvania-to-Northeast gas system.

To our knowledge, this is the first such test system that has been developed based on publicly available data for U.S. energy systems. We emphasize that our test system is meant to be reflective of the structure of the modeled systems within the northeastern U.S. and portions of Canada and is missing detail. At this stage the test system cannot (and is not meant to) reproduce observed market or system behaviors in either the power grid or the gas transmission network with a high degree of precision. The integrated gas-grid test system is, however, under continuous improvement and refinement.

The electric transmission portion of the test system is taken directly from the 36-bus NPCC model in [21], with a few modifications. At this stage of model development, our power grid model is a pure AC system, so DC elements in the 36-bus NPCC model are ignored. Rather than utilizing the locational price (bid) data provided in [21], we utilize marginal costs for coal, nuclear, hydro, wind, oil and refuse generation as reported in [22]. Our marginal cost figures for gas-fired power plants utilize average wintertime natural gas prices paid by power plants, as reported to the U.S. Energy Information Administration, for the states covered by our test system (Pennsylvania, New Jersey, New York and the six 
New England States). We translate those natural gas prices into generator marginal costs assuming a heat rate of 8,000 BTU/kWh for all gas-fired power plants in our test system. This efficiency assumption regarding gas-fired power plants will be relaxed in a future version of the test system, to allow for heterogeneity in gas turbine technology (e.g., combinedcycle versus combustion turbines). Generator marginal costs are shown in Table 6.

Table 6: Generator marginal costs (\$MWh).

\begin{tabular}{cll}
\cline { 1 - 1 } Coal & & 20.76 \\
Nuclear & & 16.50 \\
Natural Gas & & 89.36 \\
Oil & & 127.35 \\
Hydroelectric & & 10 \\
Refuse & & 50 \\
Wind & & 20 \\
\hline
\end{tabular}

We construct a representation of the interstate natural gas transmission system in the northeastern U.S. using publicly available data from the relevant gas transmission companies. Unlike the electricity sector, the gas transmission network consists of assets owned and controlled by a large number of separate entities, even within the same geographic area.

A list of data sources utilized for the construction of a gas transmission network is shown in Table 7. By and large, data from the pipeline system maps published by gas transmission companies (some of which are quite detailed) and informational postings (similar to the OASIS system utilized in the electricity sector) were utilized to determine network structure. Data gathered from these sources includes basic pipeline topology and length estimates, flow constraints, approximate locations of compressor stations, pipeline diameters, locations of off-take points for end-use consumers, interchanges with other pipeline systems, or local gas distribution companies. In some cases, data from informational postings can be used to determine withdrawal magnitudes for specific customer types. Compressor stations are modeled as branches of length zero, rather than being modeled as nodes. This allows us to determine inlet and outlet pressures at the compressor stations.

We define several gas sources in the northeastern gas transmission test system. First, gas deliveries from outside of the geographic area (e.g., from the Gulf region or from Canada) are modeled as bulk injections into the gas transmission system at our geographic boundary. For example, deliveries into the northeastern region along the Williams Transco system are modeled as injections at the Transco gas node corresponding to the Pennsylvania border. Second, the Marcellus shale is modeled as a bulk injection into the relevant gas transmission systems (Transco, Columbia and Dominion) in Pennsylvania. Third, identifiable gas storage areas (principally along the northern tier of Pennsylvania and in southern New York) are modeled as bulk injections at locations identified in pipeline system maps of the relevant transmission companies. (In other words, we model storage as equivalent to a natural gas production field and we do not consider capacity, withdrawal or replenishment constraints on gas storage, nor do we assume that withdrawals from gas storage happen in any economically optimal way.) LNG terminals in the Boston area and the
Maritimes are also modeled as supply sources. In all cases, we do not differentiate between constraints on supply sources and constraints on local pipeline capacity, i.e., we assume that any supply source in our model can produce enough to fill the pipeline to which it is directly connected.

\section{Numerical results}

As described in the previous section, our first set of results considers the problem discussed in [14] where a CEGE infrastructure is defined based on the IEEE-14 bus power system and the Belgian gas transmission network. Expansions are restricted to pipes and power lines in parallel with existing components. We use pipeline expansion costs defined in [23]. For electric power line expansion costs we consider three scenarios, (Cost Scenario 1) one where all power lines cost the same as the cheapest pipe, (Cost Scenario 2) one where all power lines cost the same as the most expensive pipe, and (Cost Scenario 3) one where they all cost the same as the average pipe. We then consider three stresses for this problem, (Stress Type 1) one where we uniformly increase the demand and production for gas, (Stress Type 2) one where we uniformly increase the demand for power and generation capacity, and (Stress Type 3) one where we simultaneously increase the demand and production for both power and gas. Since the IEEE 14-bus system does not contain line limits, we introduced line limits that were $10 \%$ more than their baseline usage. For generation costs, we also used generation costs defined in the Matpower implementation of the model (directly applying the cost coefficients to gas usage at gas-fired plants).

Our second set of results focuses on the baseline demand profile of our New England (northeastern) model. All the experiments were conducted on an 8.00GB, Intel(R) Xeon(R), 2.80GHz CPU under a 64-bit Windows-7 OS. The model for the combined electricity-gas expansion (CEGE) planning described above is solved by applying AMPL/Cplex 12.6 [24]. CPU times are provided in seconds, optimality gaps are provided when the optimal solution is not found after 24 hours of computation.

Table 8: Nomenclature used in the result Tables 9-15.

\begin{tabular}{|c|c|c|}
\hline ID & Description & Units \\
\hline$s_{f}$ & Stress factor & $\%$ \\
\hline$c_{o}$ & Operating costs & $\$ \times 10^{3}$ \\
\hline$c_{u}$ & Upgrade costs & $\$ \times 10^{6}$ \\
\hline$p_{b}$ & Pipelines built & int \\
\hline$t_{b}$ & Transmission lines built & int \\
\hline $\begin{array}{r}T_{r_{p}} \\
r_{p}\end{array}$ & $\begin{array}{l}\text { Total (real) power produced } \\
\text { (Real) power produced }\end{array}$ & MW \\
\hline & by gas-fired power generators & MW \\
\hline$g_{p}$ & Total gas production & MMSCF/d \\
\hline CPU & $\begin{array}{l}\text { Solver CPU time - Note: Optimality } \\
\text { gap }(\%) \text { is reported when reaching } \\
\text { a time limit of } 86400 \text { s }\end{array}$ & seconds \\
\hline
\end{tabular}

IEEE 14 bus and Belgian-gas network The numerical results obtained from the studies of Stress Types 1, 2 and 3 are shown in Tables 9-11 for the 14 bus/Belgian-gas network.

There are number of interesting observations to make when considering the results of these tables. First, in all cases, as 
Table 7: Data sources for the northeastern gas transmission test system.

\begin{tabular}{l}
\hline Williams (Transco and Laser) \\
Tennessee \\
Millennium \\
Dominion \\
Iroquois \\
Portland \\
Texas East \\
Algonquin \\
\hline
\end{tabular}

www.1line.williams.com
pipeline.kindermorgan.com
millenniumpipeline.com/navigates
escript.dom.com/jsp/info_post.jsp?\&company=dt
www.iroquois.com/interactive-map.asp
www.gasnom.com/ip/pngts/
www.spectraenergy.com/Operations/US-Natural-Gas-Pipelines/Texas-Eastern-Transmission/
www.spectraenergy.com/Operations/US-Natural-Gas-Pipelines/Algonquin-Gas-Transmission/

Table 9: 14-bus/Belgian-gas case: Results on Stress Type 1 (Cost Scenarios 1, 2 and 3) with induced gas demand stress.

\begin{tabular}{|c|c|c|c|c|c|c|c|c|}
\hline$s_{f}$ & $c_{o}$ & $c_{u}$ & $p_{b}$ & $t_{b}$ & $T_{r_{p}}$ & $r_{p}$ & $g_{p}$ & $\overline{\mathrm{CPU}}$ \\
\hline & \multicolumn{8}{|c|}{ Cost Scenario - 1} \\
\hline $0 \%$ & 6978.0 & 72.3 & 0 & 1 & 261.2 & 261.2 & 45.5 & 18 \\
\hline $25 \%$ & 6978.0 & 1351.7 & 1 & 1 & 261.2 & 261.2 & 56.5 & 18 \\
\hline $50 \%$ & 6978.0 & 1351.7 & 1 & 1 & 261.2 & 261.2 & 67.6 & 13 \\
\hline $75 \%$ & 6978.0 & 1691.2 & 2 & 1 & 261.2 & 261.2 & 78.6 & 14 \\
\hline \multirow[t]{2}{*}{$100 \%$} & 6978.0 & 1769.5 & 3 & 1 & 261.2 & 261.2 & 89.6 & 27 \\
\hline & \multicolumn{8}{|c|}{ Cost Scenario - 2} \\
\hline $0 \%$ & 7297.2 & 0.0 & 0 & 0 & 261.4 & 253.4 & 45.4 & 2 \\
\hline $25 \%$ & 7297.2 & 1279.5 & 1 & 0 & 261.4 & 253.4 & 56.5 & 2 \\
\hline $50 \%$ & 7297.2 & 1279.5 & 1 & 0 & 261.4 & 253.4 & 67.5 & 1 \\
\hline $75 \%$ & 7297.2 & 1618.9 & 2 & 0 & 261.4 & 253.4 & 78.6 & 3 \\
\hline \multirow[t]{2}{*}{$100 \%$} & 7297.2 & 1697.2 & 3 & 0 & 261.4 & 253.4 & 89.6 & 1 \\
\hline & \multicolumn{8}{|c|}{ Cost Scenario - 3} \\
\hline $0 \%$ & 7297.2 & 0.0 & 0 & 0 & 261.4 & 253.4 & 45.4 & 2 \\
\hline $25 \%$ & 7297.2 & 1279.5 & 1 & 0 & 261.4 & 253.4 & 56.5 & 2 \\
\hline $50 \%$ & 7297.4 & 1279.5 & 1 & 0 & 261.4 & 253.4 & 67.5 & 3 \\
\hline $75 \%$ & 7297.4 & 1618.9 & 2 & 0 & 261.4 & 253.4 & 78.6 & 2 \\
\hline \multirow[t]{2}{*}{$100 \%$} & 7297.2 & 1697.2 & 3 & 0 & 261.4 & 253.4 & 89.6 & 3 \\
\hline & \multicolumn{8}{|c|}{ Cost Scenarios - 1,2 and 3} \\
\hline $125 \%$ & inf & inf & - & - & - & - & - & 2 \\
\hline
\end{tabular}

Table 10: 14-bus/Belgian-gas case: Results on Stress Type 2 (Cost Scenarios 1, 2 and 3) with induced electric power demand stress.

\begin{tabular}{|c|c|c|c|c|c|c|c|c|}
\hline$s_{f}$ & $c_{o}$ & $c_{u}$ & $p_{b}$ & $t_{b}$ & $T_{r_{p}}$ & $r_{p}$ & $g_{p}$ & $\overline{\mathrm{CPU}}$ \\
\hline & \multicolumn{8}{|c|}{ Cost Scenario - 1} \\
\hline $0 \%$ & 6978.1 & 72.3 & 0 & 1 & 261.2 & 261.2 & 45.5 & 15 \\
\hline $25 \%$ & 9044.5 & 361.3 & 0 & 5 & 326.8 & 326.8 & 45.8 & 21 \\
\hline $50 \%$ & 10724.9 & 867.2 & 0 & 12 & 392.1 & 392.1 & 46.1 & 802 \\
\hline $75 \%$ & 13236.5 & 1300.8 & 0 & 18 & 457.4 & 457.4 & 46.5 & 37 \\
\hline \multirow{2}{*}{$100 \%$} & 15229.8 & 1878.9 & 0 & 26 & 523.1 & 504.6 & 46.7 & 32 \\
\hline & \multicolumn{8}{|c|}{ Cost Scenario - 2} \\
\hline $0 \%$ & 7297.3 & 0.0 & 0 & 0 & 261.4 & 253.4 & 45.4 & 2 \\
\hline $25 \%$ & 9127.7 & 7878.3 & 0 & 4 & 327.5 & 305.5 & 45.7 & 24 \\
\hline $50 \%$ & 11841.0 & 17726.1 & 0 & 9 & 393.8 & 329.6 & 45.8 & 155 \\
\hline $75 \%$ & 13673.3 & 27573.9 & 0 & 14 & 457.5 & 401.4 & 46.2 & 41 \\
\hline \multirow[t]{2}{*}{$100 \%$} & 16340.2 & 39391.3 & 0 & 20 & 523.4 & 438.7 & 46.4 & 27 \\
\hline & \multicolumn{8}{|c|}{ Cost Scenario - 3} \\
\hline $0 \%$ & 7297.3 & 0.0 & 0 & 0 & 261.4 & 253.4 & 45.4 & 2 \\
\hline $25 \%$ & 9127.7 & 2361.6 & 0 & 4 & 327.5 & 305.5 & 45.7 & 18 \\
\hline $50 \%$ & 10940.6 & 6494.4 & 0 & 11 & 392.7 & 381.2 & 46.1 & 137 \\
\hline $75 \%$ & 13150.5 & 9446.4 & 0 & 16 & 457.9 & 446.1 & 46.4 & 24 \\
\hline \multirow[t]{2}{*}{$100 \%$} & 15652.0 & 12988.8 & 0 & 22 & 522.7 & 486.5 & 46.6 & 23 \\
\hline & \multicolumn{8}{|c|}{ Cost Scenarios $-1,2$ and 3} \\
\hline $125 \%$ & inf & inf & - & - & - & - & - & 3 \\
\hline
\end{tabular}

the level of stress increases, the combined cost of operations and expansion increases. This is not unexpected, as intuitively, increased demand for resources increases the cost of those resources. Second, given the relatively small cost associated with the natural gas generators, they provide roughly $90 \%$ of
Table 11: 14-bus/Belgian-gas case: Results on Stress Type 3 (Cost Scenarios 1, 2 and 3) with induced electric power and gas demand stress.

\begin{tabular}{|c|c|c|c|c|c|c|c|c|}
\hline$s_{f}$ & $c_{o}$ & $c_{u}$ & $p_{b}$ & $t_{b}$ & $T_{r_{p}}$ & $r_{p}$ & $g_{p}$ & $\mathrm{CPU}$ \\
\hline & \multicolumn{8}{|c|}{ Cost Scenario - 1} \\
\hline $0 \%$ & 6978.1 & 72.3 & 0 & 1 & 261.2 & 261.2 & 45.5 & 17 \\
\hline $25 \%$ & 9044.5 & 1640.8 & 1 & 5 & 326.8 & 326.8 & 56.8 & 18 \\
\hline $50 \%$ & 10724.9 & 2146.6 & 1 & 12 & 392.1 & 392.1 & 68.2 & 675 \\
\hline $75 \%$ & 13236.7 & 2919.7 & 2 & 18 & 457.4 & 457.4 & 79.6 & 41 \\
\hline \multirow[t]{2}{*}{$100 \%$} & 15229.9 & 3576.2 & 3 & 26 & 523.1 & 504.6 & 90.9 & 40 \\
\hline & \multicolumn{8}{|c|}{ Cost Scenario - 2} \\
\hline $0 \%$ & 7297.3 & 0.0 & 0 & 0 & 261.4 & 253.4 & 45.4 & 2 \\
\hline $25 \%$ & 9127.7 & 157.7 & 1 & 4 & 327.5 & 305.5 & 56.7 & 40 \\
\hline $50 \%$ & 11841.0 & 19005.5 & 1 & 9 & 393.8 & 329.6 & 67.9 & 72 \\
\hline $75 \%$ & 13673.2 & 29192.8 & 2 & 14 & 457.5 & 401.4 & 79.3 & 36 \\
\hline \multirow[t]{2}{*}{$100 \%$} & 16340.2 & 41088.5 & 3 & 20 & 523.4 & 438.7 & 90.6 & 25 \\
\hline & \multicolumn{8}{|c|}{ Cost Scenario - 3} \\
\hline $0 \%$ & 7297.3 & 0.0 & 0 & 0 & 261.4 & 253.4 & 45.4 & 2 \\
\hline $25 \%$ & 9128.9 & 3641.1 & 1 & 4 & 327.6 & 305.7 & 56.7 & 11 \\
\hline $50 \%$ & 10940.9 & 7773.9 & 1 & 11 & 392.7 & 381.5 & 68.2 & 113 \\
\hline $75 \%$ & 13150.5 & 11065.3 & 2 & 16 & 457.9 & 446.1 & 79.6 & 20 \\
\hline \multirow[t]{2}{*}{$100 \%$} & 15651.8 & 14686.1 & 3 & 22 & 522.7 & 486.4 & 90.8 & 33 \\
\hline & \multicolumn{8}{|c|}{ Cost Scenarios $-1,2$ and 3} \\
\hline $125 \%$ & inf & inf & - & - & - & - & - & 2 \\
\hline
\end{tabular}

all power and are used at near maximum capacity. So, at the coupling points between the system, there is only flexibility to decrease interactions through reduction of gas-fired generator output.

Table 9 provides a number of interesting insights into the structure of the problem. First, the pipe solutions are not sensitive to the cost of the expansion in the power system. The power line solutions are sensitive to the cost of the power lines. In cost scenario 1 , the cost of power lines is sufficiently small so that a line is built to reduce operating costs. Second, the solutions are not able to utilize the ability to shed natural gas generator load to eliminate the need for expansion. Either it is not cost effective to do so, or the induced demands are so large that the gas generators can continue to use excess capacity in newly built pipes.

Similar observations are also seen in Table 10. In this set of problems, only electric power experiences increases in demand. Here there are no expansions performed in the natural gas network. The power system is able to ask for any gas it needs without causing problems in the gas system. Thus, the only response we see to changes in power line costs is a tradeoff between operations cost (using existing expensive generation capacity to meet new power demand) and expansion cost (build additional power lines to allow more cheap generation to be used). We see a similar effect in 
Table 12: Induced electric power demand stress on the integrated 14 bus/Belgian gas network for varying $\beta$ values: $\{0.2,0.4,0.6,0.8\}$ (Cost Scenario - 1).

\begin{tabular}{|c|c|c|c|c|c|c|c|c|}
\hline$s_{f}$ & $c_{o}$ & $c_{u}$ & $p_{b}$ & $t_{b}$ & $T_{r_{p}}$ & $r_{p}$ & $g_{p}$ & $\mathrm{CPU}$ \\
\hline & \multicolumn{8}{|c|}{$\beta=0.2$} \\
\hline $0 \%$ & 7297.5 & 0.0 & 0 & 0 & 261.4 & 253.4 & 45.4 & 1 \\
\hline $5 \%$ & 7479.0 & 72.3 & 0 & 1 & 274.2 & 274.2 & 45.5 & 7 \\
\hline $10 \%$ & 7984.0 & 144.5 & 0 & 2 & 287.2 & 287.2 & 45.6 & 4 \\
\hline $25 \%$ & 9044.5 & 361.3 & 0 & 5 & 326.8 & 326.8 & 45.8 & 8 \\
\hline $50 \%$ & 10941.0 & 794.9 & 0 & 11 & 392.7 & 381.5 & 46.1 & 138 \\
\hline $75 \%$ & 13150.5 & 1156.3 & 0 & 16 & 457.9 & 446.1 & 46.4 & 18 \\
\hline \multirow[t]{2}{*}{$100 \%$} & 15353.0 & 1662.1 & 0 & 23 & 523.2 & 488.1 & 46.6 & 12 \\
\hline & \multicolumn{8}{|c|}{$\beta=0.4$} \\
\hline $0 \%$ & 6978.0 & 72.3 & 0 & 1 & 261.2 & 261.2 & 45.5 & 10 \\
\hline $5 \%$ & 7479.3 & 72.3 & 0 & 1 & 274.2 & 274.2 & 45.5 & 8 \\
\hline $10 \%$ & 7984.3 & 144.5 & 0 & 2 & 287.2 & 287.2 & 45.6 & 4 \\
\hline $25 \%$ & 9044.5 & 361.3 & 0 & 5 & 326.8 & 326.8 & 45.8 & 22 \\
\hline $50 \%$ & 10724.8 & 867.2 & 0 & 12 & 392.1 & 392.1 & 46.1 & 249 \\
\hline $75 \%$ & 13236.5 & 1300.8 & 0 & 18 & 457.4 & 457.4 & 46.5 & 27 \\
\hline \multirow[t]{2}{*}{$100 \%$} & 15229.8 & 1878.9 & 0 & 26 & 523.1 & 504.6 & 46.7 & 10 \\
\hline & \multicolumn{8}{|c|}{$\beta=0.6$} \\
\hline $0 \%$ & 6978.2 & 72.3 & 0 & 1 & 261.2 & 261.2 & 45.5 & 8 \\
\hline $5 \%$ & 7479.2 & 72.3 & 0 & 1 & 274.2 & 274.2 & 45.5 & 8 \\
\hline $10 \%$ & 7984.2 & 144.5 & 0 & 2 & 287.2 & 287.2 & 45.6 & 4 \\
\hline $25 \%$ & 9044.5 & 361.3 & 0 & 5 & 326.8 & 326.8 & 45.8 & 13 \\
\hline $50 \%$ & 10724.8 & 867.2 & 0 & 12 & 392.1 & 392.1 & 46.1 & 193 \\
\hline $75 \%$ & 13236.7 & 1300.8 & 0 & 18 & 457.4 & 457.4 & 46.5 & 20 \\
\hline \multirow[t]{2}{*}{$100 \%$} & 15209.2 & 1951.2 & 0 & 27 & 523.0 & 506.4 & 46.7 & 17 \\
\hline & \multicolumn{8}{|c|}{$\beta=0.8$} \\
\hline $0 \%$ & 6978.1 & 72.3 & 0 & 1 & 261.2 & 261.2 & 45.5 & 8 \\
\hline $5 \%$ & 7479.1 & 72.3 & 0 & 1 & 274.2 & 274.2 & 45.5 & 8 \\
\hline $10 \%$ & 7984.3 & 144.5 & 0 & 2 & 287.2 & 287.2 & 45.6 & 3 \\
\hline $25 \%$ & 9044.5 & 361.3 & 0 & 5 & 326.8 & 326.8 & 45.8 & 8 \\
\hline $50 \%$ & 10724.9 & 867.2 & 0 & 12 & 392.1 & 392.1 & 46.1 & 199 \\
\hline $75 \%$ & 13236.5 & 1300.8 & 0 & 18 & 457.4 & 457.4 & 46.5 & 38 \\
\hline \multirow[t]{2}{*}{$100 \%$} & 15120.5 & 2095.7 & 0 & 29 & 522.7 & 506.3 & 46.7 & 21 \\
\hline & \multicolumn{8}{|c|}{$\forall \beta$ values \& Cost Scenario - 1} \\
\hline $125 \%$ & inf & inf & - & - & - & - & - & 2 \\
\hline
\end{tabular}

Table 11 where there is insensitivity in the gas system solutions to changes in the power system expansion costs.

Finally, the computational requirements for solving these problems are modest. The CPU requirement is no more than 3 minutes in 43 out of 45 instances (typically much smaller). IEEE 14 bus and Belgian-gas network: cost variance Here, we consider electric power demand stress experiments (Scenario Type 2) on the integrated IEEE 14 bus and Belgiangas network for $\beta=\{0.2,0.4,0.6,0.8\}$. There are two gasfired plants (nodes 2 and 3 ) that are coupled to gas nodes 4 and 12, respectively (see Fig.2). We modified the generation cost so that generator 2 is cheaper than generator 3 . We also artificially tightened the pressure lower bound at gas node 4 . As are in seen in these results, the solution changes as a function of $\beta$, i.e. the solution begins to favor adding additional power lines to take advantage of cheaper generation cost at generator 2 .

New England case In this section we present the results on the integrated 36-bus/Pennsylvania-To-Northeast network (Tables 13-16) based on each stress type and cost scenario as described in the previous section. In all cases $\beta=0.5$. This is a large scale instance; however, solutions (with optimality gaps) are obtained within the one day time limit imposed on the computation.
Table 13: New England case: Results on Stress Type 1 (Cost Scenarios 1, 2 and 3) with induced gas demand stress.

\begin{tabular}{|c|c|c|c|c|c|c|c|c|}
\hline$s_{f}$ & $c_{o}$ & $c_{u}$ & $p_{b}$ & $t_{b}$ & $T_{r_{p}}^{(*)}$ & $r_{p}^{(*)}$ & $g_{p}$ & $\mathrm{CPU}$ \\
\hline & \multicolumn{8}{|c|}{ Cost Scenario - 1} \\
\hline $0 \%$ & 11078.6 & 0.0 & 0 & 0 & 140.7 & 119.6 & 96.4 & 43 \\
\hline $200 \%$ & 11078.0 & 0.0 & 0 & 0 & 140.7 & 119.6 & 267.4 & 20137 \\
\hline $225 \%$ & 11078.0 & 475.0 & 6 & 0 & 140.7 & 119.6 & 288.8 & $99 \%$ \\
\hline $250 \%$ & 11053.9 & 391.4 & 7 & 0 & 142.2 & 119.1 & 312.9 & $94 \%$ \\
\hline $275 \%$ & 11078.0 & 739.4 & 7 & 0 & 140.7 & 119.6 & 331.5 & $89 \%$ \\
\hline \multirow[t]{2}{*}{$300 \%$} & 9433.9 & 1097.9 & 14 & 0 & 141.9 & 86.9 & 350.8 & $86 \%$ \\
\hline & \multicolumn{8}{|c|}{ Cost Scenario - 2} \\
\hline $0 \%$ & 11078.6 & 0.0 & 0 & 0 & 140.7 & 119.6 & 96.4 & 176 \\
\hline $200 \%$ & 11078.0 & 412.7 & 5 & 0 & 140.7 & 119.6 & 267.4 & $99 \%$ \\
\hline $225 \%$ & 10392.6 & 121.7 & 3 & 0 & 141.8 & 110.4 & 290.5 & 50574 \\
\hline $250 \%$ & 11109.6 & 630.2 & 10 & 0 & 141.1 & 120.0 & 311.3 & $98 \%$ \\
\hline $275 \%$ & 250.4 & 718.3 & 9 & 0 & 141.6 & 55.6 & 323.3 & $94 \%$ \\
\hline \multirow[t]{2}{*}{$300 \%$} & 11137.0 & 1003.0 & 12 & 0 & 141.4 & 120.3 & 353.5 & $86 \%$ \\
\hline & \multicolumn{8}{|c|}{ Cost Scenario - 3} \\
\hline $0 \%$ & 11078.6 & $\overline{0.0}$ & 0 & 0 & 140.7 & 119.6 & 96.4 & 108 \\
\hline $200 \%$ & 11084.8 & 0.0 & 0 & 0 & 140.8 & 119.7 & 267.4 & 80801 \\
\hline $225 \%$ & 10526.0 & 158.7 & 4 & 0 & 142.4 & 112.0 & 291.3 & $99 \%$ \\
\hline $250 \%$ & 11077.9 & 577.8 & 8 & 0 & 140.7 & 119.6 & 311.0 & $99 \%$ \\
\hline $275 \%$ & 10360.0 & 850.9 & 9 & 0 & 141.7 & 110.0 & 333.4 & $92 \%$ \\
\hline \multirow[t]{2}{*}{$300 \%$} & 11077.3 & 975.9 & 12 & 0 & 140.7 & 119.6 & 352.9 & $89 \%$ \\
\hline & \multicolumn{8}{|c|}{ Cost Scenarios $-1,2$ and 3} \\
\hline $500 \%$ & inf & inf & - & - & - & - & - & 110 \\
\hline
\end{tabular}

Table 14: New England case: Results on Stress Type 2 (Cost Scenarios 1, 2 and 3) with induced electric power demand stress.

\begin{tabular}{|c|c|c|c|c|c|c|c|c|}
\hline$s_{f}$ & $c_{o}$ & $c_{u}$ & $p_{b}$ & & $T_{r_{p}}^{(*)}$ & $r_{p}^{(*)}$ & $g_{p}$ & $\mathrm{CPU}$ \\
\hline & \multicolumn{8}{|c|}{ Cost Scenario - 1} \\
\hline $0 \%$ & 11078.6 & 0.0 & 0 & 0 & 140.7 & 119.6 & 96.4 & 48 \\
\hline $30 \%$ & 14315.8 & 9.8 & 0 & 1 & 182.7 & 154.4 & 103.3 & 358 \\
\hline $40 \%$ & 15312.4 & 19.6 & 0 & 2 & 196.7 & 165.3 & 105.9 & 134 \\
\hline \multirow[t]{2}{*}{$45 \%$} & 15825.8 & 29.4 & 0 & 3 & 203.6 & 170.8 & 107.3 & 494 \\
\hline & \multicolumn{8}{|c|}{ Cost Scenario - 2} \\
\hline $0 \%$ & 11078.6 & 0.0 & 0 & 0 & 140.7 & 119.6 & 96.4 & 198 \\
\hline $30 \%$ & 14174.2 & 555.5 & 0 & 1 & 182.7 & 152.9 & 103.2 & 126 \\
\hline $40 \%$ & 15314.6 & 1110.9 & 0 & 2 & 196.8 & 165.3 & 106.2 & 145 \\
\hline \multirow[t]{2}{*}{$45 \%$} & 15813.8 & 1666.4 & 0 & 3 & 203.6 & 170.6 & 107.3 & 196 \\
\hline & \multicolumn{8}{|c|}{ Cost Scenario - 3} \\
\hline $0 \%$ & 11078.6 & 0.0 & 0 & 0 & 140.7 & 119.6 & 96.4 & 85 \\
\hline $30 \%$ & 14313.2 & 118.3 & 0 & 1 & 182.7 & 154.4 & 103.5 & 498 \\
\hline $40 \%$ & 15312.6 & 236.6 & 0 & 2 & 196.7 & 165.3 & 105.9 & 169 \\
\hline \multirow[t]{2}{*}{$45 \%$} & 15993.4 & 354.9 & 0 & 3 & 203.7 & 172.9 & 107.5 & 343 \\
\hline & \multicolumn{8}{|c|}{ Cost Scenarios $-1,2$ and 3} \\
\hline $50 \%$ & inf & inf & - & - & - & - & - & 273 \\
\hline
\end{tabular}

\section{Conclusion}

Increased penetration of natural generation in power systems has led to increased economic and reliability risk in power systems. This is particularly evident when both systems are at their coincident peaks. One mechanism for alleviating this risk relies on upgrading existing gas and power systems to accommodate increased dependencies between gas and electricity. In this paper we develop a tractable optimization for upgrading and designing joint gas and electric systems to reduce this risk. We have shown how this model is used under a variety of different planning scenarios.

There are a number of directions for future work in this area. 
Table 15: New England case: Results on Stress Type 3 (Cost Scenarios 1, 2 and 3) with induced electric power and gas demand stress.

\begin{tabular}{|c|c|c|c|c|c|c|c|c|}
\hline$s_{f}$ & $c_{o}$ & $c_{u}$ & $p_{b}$ & $t_{b}$ & $T_{r_{p}}^{(*)}$ & $r_{p}^{(*)}$ & $g_{p}$ & $\mathrm{CPU}$ \\
\hline & \multicolumn{8}{|c|}{ Cost Scenario - 1} \\
\hline $0 \%$ & 11078.6 & 0.0 & 0 & 0 & 140.7 & 119.6 & 96.4 & 49 \\
\hline $30 \%$ & 14173.8 & 9.8 & 0 & 1 & 182.7 & 152.9 & 128.9 & 87 \\
\hline $40 \%$ & 15353.2 & 19.6 & 0 & 2 & 197.1 & 165.8 & 140.4 & 170 \\
\hline \multirow[t]{2}{*}{$45 \%$} & 15324.4 & 29.4 & 0 & 3 & 205.7 & 157.9 & 140.6 & 190 \\
\hline & \multicolumn{8}{|c|}{ Cost Scenario - 2} \\
\hline $0 \%$ & 11078.6 & 0.0 & 0 & 0 & 140.7 & 119.6 & 96.4 & 197 \\
\hline $30 \%$ & 14174.2 & 555.5 & 0 & 1 & 182.7 & 152.9 & 128.9 & 381 \\
\hline $40 \%$ & 15312.6 & 1110.9 & & 2 & 196.7 & 165.3 & 140.1 & 1024 \\
\hline \multirow[t]{2}{*}{$45 \%$} & 15993.2 & 1666.4 & & 3 & 203.7 & 172.9 & 146.0 & 754 \\
\hline & \multicolumn{8}{|c|}{ Cost Scenario - 3} \\
\hline $0 \%$ & 11078.6 & 0.0 & 0 & 0 & 140.7 & 119.6 & 96.4 & 87 \\
\hline $30 \%$ & 14320.4 & 118.3 & 0 & 1 & 182.7 & 154.5 & 129.0 & 133 \\
\hline $40 \%$ & 14966.0 & 236.6 & 0 & 2 & 200.5 & 159.9 & 145.5 & 471 \\
\hline \multirow[t]{2}{*}{$45 \%$} & 15813.4 & 354.9 & 0 & 3 & 203.6 & 170.6 & 145.8 & 168 \\
\hline & \multicolumn{8}{|c|}{ Cost Scenarios - 1,2 and 3} \\
\hline $50 \%$ & inf & inf & - & - & - & - & - & 158 \\
\hline
\end{tabular}

Table 16: Results on the New England case with induced electric power and gas demands at $45 \%$ and $225 \%$ stress levels, respectively, for Cost Scenarios 1, 2 and 3 (non-uniform Stress Type 3).

\begin{tabular}{c|lrlllllr}
\hline \hline SCEN & $c_{o}$ & $c_{u}$ & $p_{b}$ & $t_{b}$ & $T_{r_{p}}^{(*)}$ & $r_{p}^{(*)}$ & $g_{p}$ & CPU \\
\hline 1 & 12968.8 & 200.2 & 2 & 3 & 205.0 & 115.6 & 288.4 & $85 \%$ \\
2 & 15990.9 & 1788.1 & 3 & 3 & 203.7 & 172.9 & 301.0 & $6 \%$ \\
3 & 16003.9 & 563.3 & 2 & 3 & 203.8 & 173.0 & 300.1 & $36 \%$ \\
\hline \multicolumn{8}{c}{$(*)$} & $10^{3}$
\end{tabular}

First, the dependencies between the systems are inherently influenced by exogenous factors such as extreme weather. It will be interesting to expand this model to include resilience criteria related to such events. Second, expansion decisions are not typically performed all at once. They are often undertaken over time in a staged approach. It will be important to extend these results to a multi stage formulation. Finally, it will be important to develop iterative approaches for refining these relaxations when solutions are obtained that are not tight.

\section{References}

[1] PLM Interconnection, "Analysis of Operational Events and Market Impacts During the January 2014 Cold Weather Events," PLM Interconnection, Tech. Rep., 2014.

[2] E. Gil, A. Quelhas, J. McCalley, and T. van Voorhis, "Modeling integrated energy transportation networks for analysis of economic efficiency and network interdependencies," White Paper, Iowa State University, 26-Nov 2004.

[3] A. Nobakht, M. Javadi, Z. Manoochehri, and S. Heidari, "Simultaneous generation expansion planning and natural gas expansion planning," ACEEE International Journal on Control System and Instrumentation, vol. 2, no. 1, pp. 33-38, 2011.

[4] G. Peters, "Gas and electric infrastructure interdependency analysis," Technical report prepared for the Midwest Independent Transmission System Operator (MISO), dba EnVision Energy Solutions, USA, Tech. Rep., 2012.

[5] R. Rubio-Barros, D. Ojeda-Esteybar, O. Año, and A. Vargas, "Combined operational planning of natural gas and electric power systems: State of the art," Chapter 12, Natural Gas, edited by Primoz Potocnik, pp. 271288, ISBN: 978-953-307-112-1, InTech 2010.
[6] — , "Integrated natural gas and electricity market: A survey of the state of the art in operation planning and market issues," 2008 IEEE/PES, Transmission and Distribution Conference and Exposition: Latin America, Bogota, Colombia, pp. 1-8, 2008.

[7] C. Borraz-Sánchez, R. Bent, S. Backhaus, H. Hijazi, and P. Van Hentenryck, "Convex relaxations for gas expansion planning," under review, 2015.

[8] H. Hijazi, C. Coffrin, and P. Van Hentenryck, "Convex quadratic relaxations for mixed-integer nonlinear programs in power systems," White paper, vol. 1, no. 1, pp. 1-31, 2015.

[9] M. Chaudry, N. Jenkins, M. Qadrdan, and J. Wu, "Combined gas and electricity network expansion planning," Applied Energy, vol. 113, pp. 1171-1187, 2014.

[10] S. An, Q. Li, and T. Gedra, "Natural gas and electricity optimal power flow," in Transmission and Distribution Conference and Exposition, IEEE PES, vol. 1, 2003, pp. 138-143.

[11] M. Chaudry, N. Jenkins, and G. Strbac, "Multi-time period gas and electricity network optimization," Electric Power Systems Research, vol. 78, pp. 1265-1279, 2008.

[12] C. Correa-Posada and P. Sánchez-Martín, "Security-constrained optimal power and natural-gas flow," IEEE Transactions on Power Systems, vol. 29 , no. 4, pp. 1780-1787, 2014.

[13] M. Damavandi, I. Kiaei, M. Sheikh-El-Eslami, and H. Seifi, "New approach to gas network modeling in unit commitment," Energy, vol. 36, pp. 6243-6250, 2011.

[14] C. Unsihuay-Vila, J. Marangon-Lima, and A. Zambroni de Souza, "Modeling the integrated natural gas and electricity optimal power flow," in Proceedings 2007 IEEE Power Engineering Society (PES) General Meeting. IEEE, 2007, pp. 24-28.

[15] C. Unsihuay-Vila, J. Marangon-Lima, A. Zambroni de Souza, I. PerezArriaga, and P. Balestrassi, "A model to long-term, multiarea, multistage, and integrated expansion planning of electricity and natural gas systems," IEEE Transactions on Power Systems, vol. 25, no. 2, pp. 1154$1168,2010$.

[16] C. Unsihuay-Vila, J. Marangon-Lima, and A. Zambroni de Souza, "Integrated power generation and natural gas expansion planning," in Proceedings 2007 IEEE/PES PowerTech Conference. IEEE, July 1-5, Lausanne, Switzerland 2007, pp. 1404-1409.

[17] F. Al-Khayyal and J. Falk, "Optimization of capacity expansion planning for gas transportation networks," Jointly constrained biconvex programming, vol. 8, no. 2, pp. 273-286, 1983.

[18] G. McCormick, "Computability of global solutions to factorable nonconvex programs, part i: convex underestimating problems," Mathematical Programming, vol. 10, pp. 147-175, 1976

[19] H. Hijazi, P. Bonami, G. Cornuéjols, and A. Ouorou, "Mixed integer nonlinear programs featuring on/off constraints: convex analysis and applications," Electronic notes in Discrete Mathematics, vol. 36, pp. 1153-1160, 2010.

[20] — "Mixed-integer nonlinear programs featuring on/off constraints," Computational Optimization and Applications, vol. 52, no. 2, pp. 537-558, 2012. [Online]. Available: http://dx.doi.org/10.1007/ s10589-011-9424-0

[21] E. Allen, J. Lang, and M. Ilić, "A combined equivalenced-electric, economic, and market representation of the northeastern power coordinating council u.s. electric power system," IEEE Transactions on Power Systems, vol. 23, no. 3, pp. 896-907, 2008.

[22] A. Newcomer, S. Blumsack, J. Apt, L. Lave, and M. Morgan, "The short run economic and environmental effects of a carbon tax on u.s. electric generation," Env. Sci. Tech., vol. 42, pp. 3139-3144, 2008.

[23] F. Babonneau, Y. Nesterov, and J.-P. Vial, "Design and operations of gas transmission networks," Operations Research, vol. 60, no. 1, pp. 34-47, January-February 2012

[24] I. ILOG CPLEX Optimization Studio, "Cplex users manual, version 12 release 6," 2013. 